\title{
Utility of Systematic Coronary Risk Evaluation (SCORE) system to predict coronary artery disease severity in low to moderate risk hypertensive patients undergoing elective coronary angiography
}

\author{
Mehmet Eyuboglu ${ }^{\circledR}$, Metin Karayakalı ${ }^{\circledR}$, Kayihan Karaman ${ }^{\circledR}$, Arif Arısoy ${ }^{\circledR}$, Atac Celık $₫$ \\ Department of Cardiology, Gaziosmanpasa University, School of Medicine, Tokat, Turkey
}

\begin{abstract}
Background: Coronary artery disease $(\mathrm{CAD})$ is the leading cause of mortality in hypertensive patients. Systematic Coronary Risk Evaluation (SCORE) is the preferred scoring system to predict future fatal cardiovascular events in hypertensive patients. However, the relationship between SCORE and coronary atherosclerosis is not well described. We aimed to investigate whether SCORE has a relationship with CAD severity in hypertensive patients, even in the absence of high risk features.

Material and methods: Four hundred and fifty-two hypertensive patients who underwent elective coronary angiography and defined as low or moderate risk according to SCORE were included into the study. Patients were divided into two groups. Patients with a SCORE $<1 \%$ were defined as low risk group, and patients with a SCORE $\geq 1 \%$ and $<5 \%$ were defined as moderate risk group. The groups were compared regarding CAD severity.

Results: The frequency of stenotic CAD and multivessel disease, and mean SYNTAX score, were significantly higher in SCORE $\geq 1 \%$, and $<5 \%$ group compared to patients with SCORE $<1 \%$. Correlation analysis revealed a significant positive moderate correlation between SCORE and SYNTAX score (Pearson's $r: 0.679, p<0.001$ ). ROC curve analysis demonstrated that a SCORE $\geq 3 \%$ predicted SYNTAX score $>22$ with a sensitivity of $75 \%$ and a specificity of $86.5 \%$ (AUC: $0.879, \mathrm{p}<0.001$ ). Furthermore, multivariate analysis demonstrated that SCORE was an independent predictor of stenotic CAD (OR: 1.616, $\mathrm{p}<0.001)$, multivessel disease (OR: 1.913, $\mathrm{p}<0.001)$, and SYNTAX score $>22$. (OR: 1.817, $\mathrm{p}<0.001)$.

Conclusion: Our results suggest that SCORE is associated with CAD severity in hypertensive patients even in the absence of high risk features. The SCORE system may be useful in further risk stratification of hypertensive patients with moderate risk features and suspected CAD.
\end{abstract}

Key words: hypertension; coronary artery disease; coronary angiography; SCORE; SYNTAX score 


\section{Introduction}

Hypertension is an important risk factor for both structural and functional damage in cardiovascular system that leads a significant increase in morbidity and mortality $[1,2]$. Atherosclerosis is the main manifestation of hypertension-related vascular injury, and coronary artery disease $(\mathrm{CAD})$ is the most dangerous consequence of hypertensive end-organ damage and is the leading cause of mortality in hypertensive patients [3, 4]. Hereby, risk stratification of hypertensive patients remains the cornerstone of the adequately prevention of future cardiovascular events. Systematic Coronary Risk Evaluation (SCORE) system is a validated scoring system that predicts the 10-year risk of a first fatal atherosclerotic cardiovascular event, in relation to age, gender, smoking, total cholesterol, and systolic blood pressure (SBP) [5]. European guidelines strongly recommend to use SCORE system for cardiovascular risk assessment of hypertensive patients except those who are at high or very high risk due to known cardiovascular disease (CVD) or associated conditions $[4,6]$. However, SCORE only predicts the 10-year risk of a first fatal atherosclerotic cardiovascular event, and its association with CAD and CAD severity in low and moderate risk hypertensive patients remains unclear. Since the CAD is the leading cause of the death in hypertensive patients, definition of association of SCORE with CAD severity may provide useful information for further risk assessment in clinical practice, in particular, in hypertensive patients with low and moderate risk.

SYNTAX score is an angiographic tool that is calculated based on anatomical location and potential functional importance of coronary lesions and is used to estimate the extend, complexity and severity of CAD [7]. SYNTAX score is the cornerstone of the classification of CAD severity and is an important prognostic factor in patients with CAD [8]. Therefore, in the present study, the association of SCORE system with CAD severity in hypertensive patients who don't have high risk features was investigated. We mainly aimed to investigate whether SCORE has a relationship with SYNTAX score in hypertensive patients classified as being at low and moderate risk according to SCORE system and undergoing elective coronary angiography.

\section{Material and methods}

\section{Study design}

A total of 525 consecutive hypertensive patients who were between the age 40 and 65 and at low or moderate risk according to SCORE system, and underwent elective diagnostic coronary angiography from January 2015 to November 2019 were enrolled. Among these patients, 73 patients (patients with left ventricular hypertrophy (LVH), microalbuminuria, and retinopathy) were excluded due to presence of asymptomatic hypertension-mediated organ damage that increases the cardiovascular risk independently. As a consequence, the remaining 452 hypertensive patients were included into the study. Since the SCORE system is validated for the subjects between the age 40 and 65, patients younger than 40 years and older than 65 years were not screened for the study. Patients with established CVD or its equivalent or at high (SCORE $\geq 5 \%$ and $<10 \%$ ) or very high (SCORE $\geq 10 \%$ ) risk were also excluded at baseline and were not screened for the study.

Hypertension was defined as SBP $\geq 140 \mathrm{~mm} \mathrm{Hg}$ and/or diastolic blood pressure $\geq 90 \mathrm{~mm} \mathrm{Hg}$ in repeated measurements, and/or known treatment with antihypertensive medications. Active smoking was defined as the current regular use of cigarettes. All patients were evaluated with a detailed anamnesis, cardiac and systemic physical examination, electrocardiography and echocardiography. Laboratory analyses were performed from venous blood samples obtained after an overnight fasting. The study was conducted in full accordance with the Declaration of Helsinki and approved by the local ethics committee.

\section{Systematic Coronary Risk Evaluation (SCORE)}

SCORE is the preferred scoring system to estimate the 10-year risk of a first fatal atherosclerotic cardiovascular event, in relation to age, gender, smoking, total cholesterol, and SBP. Cardiovascular risk assessment with the SCORE system is strongly recommended for all hypertensive patients who are not already at high or very high risk due to manifest overt CVD or chronic kidney disease, or diabetes mellitus, or a markedly elevated single risk factor or $\mathrm{LVH}[4,5]$. Cardiovascular risk is analyzed in four groups based on SCORE. A calculated SCORE $\geq 10 \%$ indicates patients at very high risk, a SCORE $\geq 5 \%$ and $\leq 10 \%$ indicates patients at high risk, a SCORE $\geq 1 \%$ and $<5 \%$ indicates patients at moderate risk, and a SCORE $<1 \%$ indicates patients at low risk [4-6]. SCORE can be easily calculated from "SCORE charts" for men and women, separately. There are two suggested SCORE charts for low risk and high risk countries, respectively. Since the study conducted in Turkey, we used the "SCORE — European High 
Risk Chart" for calculation of SCORE [6]. In the present study, we divided the study population into two groups according to SCORE system. Patients with a calculated SCORE $<1 \%$ were assigned to the low risk, and patients with SCORE $\geq 1 \%$ and $<5 \%$ were assigned to the moderate risk group.

\section{Coronary angiography and SYNTAX score}

All patients underwent coronary angiography in elective conditions due to positive or suspected result of non-invasive tests. The classification of CAD was made based on coronary angiography results. Presence of $\geq 50 \%$ obstruction in any coronary artery was defined as stenotic CAD. Number of stenotic vessels and SYNTAX score were used to estimate the extend and severity of CAD. The presence of CAD was categorized as stenotic CAD (one vessel), or multivessel disease (MVD) according to the number of vessel with $\geq 50 \%$ stenosis.

SYNTAX score is calculated for the vessels with a diameter $>1.5 \mathrm{~mm}$ and with a stenosis $\geq 50 \%$, and estimates the extend, complexity and severity of $\mathrm{CAD}$ using various anatomical and functional parameters [7, 8]. Also, SYNTAX score is a predictor of adverse events in patients with CAD, and while a SYNTAX score $\leq 22$ indicates low risk patients for cardiovascular events, a SYNTAX score $>22$ defines the intermediate to high risk patients $[8,9]$. Therefore, in the present study, SYNTAX score was calculated for all vessels $>1.5 \mathrm{~mm}$ diameter and with a stenosis $\geq 50 \%$, according to SYNTAX score calculator (www.syntaxscore.com). All digital coronary angiograms were evaluated by two independent experienced cardiologists. In case of disagreement, the final decision was achieved by consensus.

\section{Statistical analysis}

Continuous and categorical variables were expressed as mean \pm standard deviation and percentages, respectively. The categorical variables were compared with the chi-square test or Fisher's exact test. The normality distribution of continuous variables was tested with Kolmogorov-Smirnov test. Continuous variables between the two groups were compared with Student's t test. Correlation between SCORE and SYNTAX score was assessed with Pearson's correlation coefficient. Receiver operating characteristics (ROC) curve analysis was performed to determine the area under the curve, and cutoff value of SCORE for predicting SYNTAX score $>22$. Multivariate logistic regression analysis was performed to define the independent predictors of stenotic CAD, MVD and SYNTAX score $>22$. A p-value of $<0.05$ was considered to indicate statistical significance.

\section{Results}

Among study population, 171 patients had a SCORE $<1 \%$, and 281 patients had a SCORE $\geq 1 \%$, and $<5 \%$. Due to the nature of the SCORE system, the frequency of women $(80.7 \%)$ were higher in patients with SCORE $<1 \%$ whereas, the frequency of men $(68 \%)$ were higher in the SCORE $\geq 1 \%$ and $<5 \%$ patient groups. In the same sense, patients with SCORE $\geq 1 \%$, and $<5 \%$ were older and had higher blood pressure levels compared to patients with SCORE $<1 \%$. Also, dyslipidemia was more frequent in SCORE $\geq 1 \%$, and $<5 \%$ group. Importantly, the frequency of stenotic CAD and multivessel disease, and mean SYNTAX score, were significantly higher in SCORE $\geq 1 \%$, and $<5 \%$ group compared to patients with $\mathrm{SCORE}<1 \%$. Table 1 demonstrates the clinical, laboratory, and angiographic characteristics of patients according to SCORE system. When comparing the patients according to SYNTAX score, patients with SYNTAX score $>22$ were older, and had higher blood pressure levels compared to patients with SYNTAX score $\leq 22$. Moreover, the mean SCORE was significantly higher in the group of patients with SYNTAX score $>22$ compared to patients with SYNTAX score $\leq 22(3.48 \pm 1.26$ vs. $1.75 \pm 1.38, \mathrm{p}<0.001)$. Table 2 shows the characteristics of patients according to SYNTAX score. Correlation analysis revealed a significant positive moderate correlation between SCORE and SYNTAX score (Pearson's r: 0.679, $\mathrm{p}<0.001$ ). Also, ROC curve analysis demonstrated that a SCORE $\geq 3 \%$ predicted SYNTAX score $>22$ with a sensitivity $75 \%$ and a specificity $86.5 \%$ [area under the curve (AUC): 0.879, $\mathrm{p}<0.001$ ] (Fig. 1)

Furthermore, multivariate logistic regression analysis demonstrated that SCORE was an independent predictor of stenotic CAD [odds ratio (OR): 1.616, 95\% confidence interval (CI): 1.055-1.901, $\mathrm{p}<0.001$ ], multivessel disease (OR: 1.913, 95\% CI: 1.545-2.091, $\mathrm{p}<0.001$ ), and SYNTAX score $>22$ (OR: 1.817, 95\% CI: 1.438-2.253, p < 0.001). Table 3 shows the independent predictors of stenotic CAD, multivessel disease and SYNTAX score $>22$ in multivariate analysis.

\section{Discussion}

The main finding of the present article was that the SCORE may be useful to predict patients with more severe $\mathrm{CAD}$ and patients at relatively high risk for future cardiovascular events, even in the absence of high risk features according to SCORE system. 
Table 1. Clinical, laboratory, and angiographic characteristics of patients according to Systematic Coronary Risk Evaluation (SCORE)

\begin{tabular}{|c|c|c|c|}
\hline & $\begin{array}{c}\text { SCORE }<1 \% \\
(n=171)\end{array}$ & $\begin{array}{c}\text { SCORE } \geq 1 \% \text { and }<5 \% \\
(\mathbf{n}=\mathbf{2 8 1 )}\end{array}$ & $\mathbf{p}$ \\
\hline Age (years) & $44.5 \pm 2.39$ & $52.5 \pm 5.51$ & $<0.001$ \\
\hline \multicolumn{4}{|l|}{ Gender } \\
\hline $\begin{array}{l}\text { Male n (\%) } \\
\text { Female } \mathrm{n}(\%)\end{array}$ & $\begin{array}{c}33(19.3 \%) \\
138(80.7 \%)\end{array}$ & $\begin{array}{c}191(68 \%) \\
90(32 \%)\end{array}$ & $<0.001$ \\
\hline Smoking n (\%) & $32(18.7 \%)$ & $108(38.4 \%)$ & $<0.001$ \\
\hline LVEF (\%) & $62.2 \pm 3.76$ & $61.9 \pm 3.78$ & 0.375 \\
\hline SBP $[\mathrm{mm} \mathrm{Hg}]$ & $143.5 \pm 9.07$ & $149 \pm 12.5$ & $<0.001$ \\
\hline $\mathrm{DBP}[\mathrm{mm} \mathrm{Hg}]$ & $84.5 \pm 7.86$ & $89.6 \pm 6.93$ & $<0.001$ \\
\hline WBC count $\left[\times 10^{3} / \mu \mathrm{L}\right]$ & $7.55 \pm 1.39$ & $7.62 \pm 1.34$ & 0.867 \\
\hline Platelet count $\left[\times 10^{3} / \mu \mathrm{L}\right]$ & $248 \pm 58.7$ & $258 \pm 62.9$ & 0.109 \\
\hline Hemoglobin $[\mathrm{g} / \mathrm{dL}]$ & $12.1 \pm 1.52$ & $13.4 \pm 1.94$ & $<0.001$ \\
\hline Creatinine [mg/dL] & $0.8 \pm 0.14$ & $0.8 \pm 0.15$ & 0.122 \\
\hline AST [U/L] & $21.9 \pm 8.61$ & $22.6 \pm 8.29$ & 0.741 \\
\hline Total cholesterol [mg/dL] & $183 \pm 35.8$ & $211 \pm 38.4$ & $<0.001$ \\
\hline LDL-cholesterol [mg/dL] & $93.6 \pm 21$ & $118 \pm 34.2$ & $<0.001$ \\
\hline HDL-cholesterol [mg/dL] & $45.2 \pm 6.89$ & $38.6 \pm 8$ & $<0.001$ \\
\hline Stenotic CAD & $60(35.1 \%)$ & $159(56.6 \%)$ & $<0.001$ \\
\hline Multivessel disease & $16(9.4 \%)$ & $74(26.3 \%)$ & $<0.001$ \\
\hline SYNTAX score & $5.18 \pm 7.07$ & $13.5 \pm 9.32$ & $<0.001$ \\
\hline
\end{tabular}

LVEF — left ventricular ejection fraction; SBP — systolic blood pressure; DBP — diastolic blood pressure; WBC — white blood cell; AST — aspartate aminotransferase; LDL — low-density lipoprotein; $\mathrm{HDL}$ — high-density lipoprotein; CAD — coronary artery disease

Table 2. Patients' characteristics according to SYNTAX score

\begin{tabular}{|c|c|c|c|}
\hline & $\begin{array}{c}\text { SYNTAX score } \leq 22 \\
(\mathrm{n}=155)\end{array}$ & $\begin{array}{c}\text { SYNTAX score }>22 \\
(n=64)\end{array}$ & $\mathbf{p}$ \\
\hline Age [y] & $51.4 \pm 6.33$ & $53.9 \pm 5.08$ & 0.005 \\
\hline \multicolumn{4}{|l|}{ Gender } \\
\hline $\begin{array}{l}\text { Male } \mathrm{n}(\%) \\
\text { Female } \mathrm{n}(\%)\end{array}$ & $\begin{array}{l}76[49 \%] \\
79[51 \%]\end{array}$ & $\begin{array}{l}50(78.1 \%) \\
14(21.9 \%)\end{array}$ & $<0.001$ \\
\hline SCORE (\%) & $1.75 \pm 1.38$ & $3.48 \pm 1.26$ & $<0.001$ \\
\hline Smoking n (\%) & $60(38.7 \%)$ & $24(37.5 \%)$ & 0.867 \\
\hline LVEF (\%) & $62.6 \pm 4.04$ & $61.8 \pm 2.04$ & 0.325 \\
\hline $\mathrm{SBP}[\mathrm{mm} \mathrm{Hg}]$ & $151 \pm 9.84$ & $158 \pm 10.04$ & $<0.001$ \\
\hline $\mathrm{DBP}[\mathrm{mm} \mathrm{Hg}]$ & $91.9 \pm 5.75$ & $97 \pm 5.47$ & $<0.001$ \\
\hline WBC count $\left[\times 10^{3} / \mu \mathrm{L}\right]$ & $8.09 \pm 1.50$ & $7.92 \pm 1.02$ & 0.127 \\
\hline AST [U/L] & $19.3 \pm 9.06$ & $21.5 \pm 5.8$ & 0.078 \\
\hline Platelet count $\left[\times 10^{3} / \mu \mathrm{L}\right]$ & $235 \pm 46.4$ & $284 \pm 84.8$ & $<0.001$ \\
\hline Hemoglobin $[\mathrm{g} / \mathrm{dL}]$ & $12.8 \pm 1.82$ & $14.4 \pm 1.42$ & $<0.001$ \\
\hline Creatinine [mg/dL] & $0.8 \pm 0.13$ & $0.8 \pm 0.14$ & 0.181 \\
\hline Total cholesterol [mg/dL] & $212 \pm 35.2$ & $226 \pm 49.2$ & 0.019 \\
\hline LDL-cholesterol [mg/dL] & $117 \pm 26.9$ & $134 \pm 48.4$ & $<0.001$ \\
\hline HDL-cholesterol [mg/dL] & $38.8 \pm 7.12$ & $33 \pm 6.22$ & $<0.001$ \\
\hline
\end{tabular}

SCORE — Systematic Coronary Risk Evaluation; LVEF — left ventricular ejection fraction; SBP — systolic blood pressure; DBP — diastolic blood pressure; WBC — white blood cell; AST — aspartate aminotransferase; LDL - low-density lipoprotein; HDL — high-density lipoprotein 


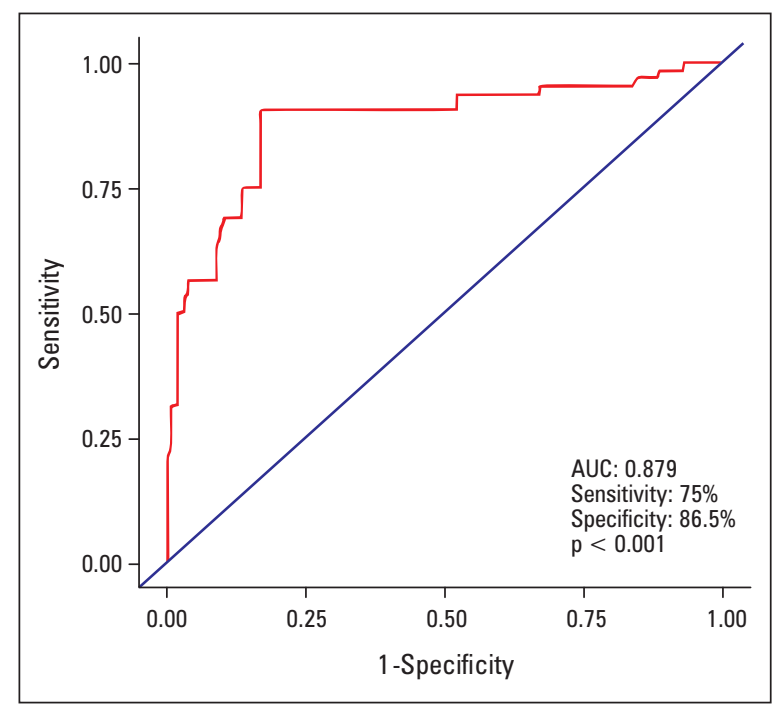

Figure 1. Receiver operating characteristic (ROC) curve analysis of Systematic Coronary Risk Evaluation (SCORE) to predict SYNTAX score $>22$

Therefore, the results of our study may be helpful for further risk stratification of hypertensive patients, in particular, for patients without high risk features and with suspected CAD. To our knowledge, this is the first study to report an independent relationship between SCORE and CAD severity in hypertensive patients with low and moderate risk based on SCORE system.

There is a consistent link between increased blood pressure and coronary atherosclerosis, and hypertension is the most important modifiable risk factor for development and progression of $\mathrm{CAD}$, from endothelial dysfunction to symptomatic obstructive CAD $[10,11]$. Atherosclerosis is the leading cause of morbidity and mortality in hypertensive patients; how- ever, the hypertension related coronary atherosclerosis generally requires a silent long process, and once $\mathrm{CAD}$ is emerged, it affects prognosis independently $[4,12]$. Hereby, early and accurate risk stratification remains the cornerstone of the implementation of preventive treatments in hypertensive subjects.

SCORE system is a useful tool in clinical practice considering multiple traditional cardiovascular risk factors, and is essential in the risk stratification of hypertensive patients [4, 6]. Nevertheless, the SCORE system mainly estimates only 10 -year risk of a first fatal atherosclerotic cardiovascular event, and its predictive value regarding non-fatal events is not well described. However, due to the nature of the SCORE system consisting five important risk factors for $\mathrm{CAD}$, it is reasonable to consider that it may be associated with various cardiovascular end points, and may be more helpful than expected in clinical practice.

Since the combination of the traditional cardiovascular risk factors predicts atherosclerosis more accurately, risk scores play an essential role in the estimation of total cardiovascular risk and subsequent implementation of preventive managements [13]. Cardiovascular risk scores including SCORE system have been shown to be associated with CAD severity in small studies conducted in unselected patients underwent coronary angiography, mainly due to high risk features [14-16]. However, no study investigated the association of SCORE system with CAD severity in isolated hypertensive patients with low and moderate risk. We determined a significant positive correlation between SCORE and CAD severity, and also found that hypertensive patients at moderate risk had significantly more severe CAD compared to those at low risk according to SCORE

Table 3. Multivariate logistic regression analysis for independent predictors of stenotic coronary artery disease (CAD), multivessel disease and SYNTAX score $>22$

\begin{tabular}{|c|c|c|c|}
\hline Variable & OR & $95 \% \mathrm{Cl}$ & p \\
\hline \multicolumn{4}{|l|}{ Stenotic CAD } \\
\hline SCORE & 1.616 & $1.055-1.901$ & $<0.001$ \\
\hline LDL-cholesterol & 1.162 & $1.034-1.290$ & $<0.001$ \\
\hline DBP & 1.068 & $1.040-1.130$ & $<0.001$ \\
\hline \multicolumn{4}{|c|}{ Multivessel disease } \\
\hline SCORE & 1.913 & $1.545-2.091$ & $<0.001$ \\
\hline LDL-cholesterol & 1.171 & $1.098-1.570$ & $<0.001$ \\
\hline \multicolumn{4}{|c|}{ SYNTAX score > 22} \\
\hline SCORE & 1.817 & $1.438-2.253$ & $<0.001$ \\
\hline LDL-cholesterol & 1.079 & $1.037-1.492$ & $<0.001$ \\
\hline
\end{tabular}


system. Hereby, our results point out the importance of further risk assessment of hypertensive patients, even if they are considered as low or, in particular, moderate risk according to SCORE system.

The main clinical importance of risk assessment by SCORE is its essential role in the providing successful prevention of future events $[4,6]$. Primary prevention methods including drug treatment reduce major cardiovascular events in patients at high risk or very high risk according to SCORE (SCORE $\geq 5 \%$ ) $[6,17]$. Hence, these patients generally receive optimal preventive treatments without need for further risk assessment. However, preventive treatment with drugs are not generally recommended for patients at low to moderate risk (SCORE $<5 \%$ ), and these patients are usually offered lifestyle advice to maintain their low to moderate risk status [6]. On the other hand, the findings of the present article suggest that a selected subgroup of hypertensive patients at moderate risk according to SCORE system may be considered as patients at relatively high risk and may provide more benefit from preventive treatments. Consequently, widening the preventive methods for hypertensive patients with suspected CAD and at moderate risk according to SCORE system may be useful for an optimal primary prevention. Nevertheless, there is lack of data regarding the clinical benefit of preventive treatments in moderate risk hypertensive patients, and future studies investigating this topic are necessary. In this sense, the key point of the present study is that SCORE may be useful for further risk stratification in hypertensive patients even in the absence of high risk features.

The present study has some limitations. First, the study included a selected patient population between the age 40 and 65 . Therefore, it is difficult to generalize our findings to hypertensive patients that are out of this age range. However, the SCORE system is validated for hypertensive subjects between the age 40 and 65, and this age range includes the vast majority of patients that will benefit from the preventive treatments. Second, although we excluded the patients with asymptomatic hypertension-mediated organ damage, there may be still some other clinical factors that increase the patients' risk independently.

\section{Conclusions}

The SCORE system may be useful in further risk stratification of hypertensive patients who have moderate risk features and are suspected of CAD. Our study suggests that SCORE has an independent relationship and positive correlation with SYN-
TAX score, in particular in patients at moderate risk, which may affect the prognosis independently. Therefore, SCORE may be useful in the discrimination of hypertensive patients at relatively high risk even if they are assigned as moderate risk according to SCORE system. Further risk assessment in this selected subgroup of hypertensive patients with suspected CAD may lead a more adequate preventive program and treatment.

\section{Conflict of interest}

Nothing to declare - we have no commercial, financial, and other relationships in any way related to the subject of this article that might create any potential conflict of interest.

Nothing to declare.

\section{Funding and support}

\section{References}

1. Kannel W. Blood pressure as a cardiovascular risk factor: prevention and treatment. JAMA. 1996; 275(20): 1571-1576, doi: 10.1001/ jama.1996.03530440051036, indexed in Pubmed: 8622248.

2. Lewington S, Clarke R, Qizilbash N, et al. Prospective Studies Collaboration. Age-specific relevance of usual blood pressure to vascular mortality: a meta-analysis of individual data for one million adults in 61 prospective studies. Lancet. 2002; 360(9349): 1903-1913, doi: 10.1016/s0140-6736(02)11911-8, indexed in Pubmed: 12493255.

3. Schmieder RE. End organ damage in hypertension. Dtsch Arztebl Int. 2010; 107(49): 866-873, doi: 10.3238/arztebl.2010.0866, indexed in Pubmed: 21191547.

4. Williams B, Mancia G, Spiering W, et al. Authors/Task Force Members:, ESC Scientific Document Group . 2018 ESC/ESH Guidelines for the management of arterial hypertension. Eur Heart J. 2018; 39(33): 3021-3104, doi: 10.1093/eurheartj/ehy339, indexed in Pubmed: 30165516.

5. Conroy RM, Pyörälä K, Fitzgerald AP, et al. SCORE project group. Estimation of ten-year risk of fatal cardiovascular disease in Europe: the SCORE project. Eur Heart J. 2003; 24(11): 987-1003, doi: 10.1016/s0195-668x(03)00114-3, indexed in Pubmed: 12788299 .

6. Piepoli MF, Hoes AW, Agewall S, et al. Authors/Task Force Members:, Authors/Task Force Members, Additional Contributor: Simone Binno (Italy), Document Reviewers:, ESC Scientific Document Group. 2016 European Guidelines on cardiovascular disease prevention in clinical practice: The Sixth Joint Task Force of the European Society of Cardiology and Other Societies on Cardiovascular Disease Prevention in Clinical Practice (constituted by representatives of 10 societies and by invited experts)Developed with the special contribution of the European Association for Cardiovascular Prevention \& Rehabilitation (EACPR). Eur Heart J. 2016; 37(29): 2315-2381, doi: 10.1093/eurheartj/ehw106, indexed in Pubmed: 27222591.

7. Sianos G, Morel MA, Kappetein AP, et al. The SYNTAX Score: an angiographic tool grading the complexity of coronary artery disease. EuroIntervention. 2005; 1(2): 219-227, indexed in Pubmed: 19758907.

8. Neumann FJ, Sousa-Uva M, Ahlsson A, et al. ESC Scientific Document Group, ESC Scientific Document Group. Considerations for the choice between coronary artery bypass grafting and percutaneous coronary intervention as revascularization strategies in major categories of patients with stable multivessel coronary artery disease: an accompanying article of the task force of the $2018 \mathrm{ESC} /$ 
EACTS guidelines on myocardial revascularization. Eur Heart J. 2019; 40(2): 204-212, doi: 10.1093/eurheartj/ehy532, indexed in Pubmed: 30165435.

9. Serruys PW, Onuma Y, Garg S, et al. Assessment of the SYNTAX score in the Syntax study. EuroIntervention. 2009; 5(1): 50-56, doi: 10.4244/eijv5i1a9, indexed in Pubmed: 19577983.

10. McInnes GT. Hypertension and coronary artery disease: cause and effect. J Hypertens Suppl. 1995; 13(2): S49-S56, doi: 10.1097/00004872-199508001-00008, indexed in Pubmed: 8576788.

11. Escobar E. Hypertension and coronary heart disease. J Hum Hypertens. 2002; 16(S1): S61-S63, doi: 10.1038/sj.jhh.1001345, indexed in Pubmed: 11986897.

12. Weber T, Lang I, Zweiker R, et al. Hypertension and coronary artery disease: epidemiology, physiology, effects of treatment, and recommendations : A joint scientific statement from the Austrian Society of Cardiology and the Austrian Society of Hypertension. Wien Klin Wochenschr. 2016; 128(13-14): 467-479, doi: 10.1007/s00508016-0998-5, indexed in Pubmed: 27278135.

13. Cooney MT, Dudina AL, Graham IM. Value and limitations of existing scores for the assessment of cardiovascular risk: a review for clinicians. J Am Coll Cardiol. 2009; 54(14): 12091227, doi: 10.1016/j.jacc.2009.07.020, indexed in Pubmed: 19778661.

14. Tolunay $\mathrm{H}$, Kurmus $\mathrm{O}$. Comparison of coronary risk scoring systems to predict the severity of coronary artery disease using the SYNTAX score. Cardiol J. 2016; 23(1): 51-56, doi: 10.5603/CJ.a2015.0074, indexed in Pubmed: 26503075.

15. Yalcin M, Kardesoglu E, Aparci M, et al. Cardiovascular risk scores for coronary atherosclerosis. Acta Cardiol. 2012; 67(5): 557-563, doi: 10.1080/ac.67.5.2174130, indexed in Pubmed: 23252006.

16. Lee BC, Lee WJ, Hsu HC, et al. Using clinical cardiovascular risk scores to predict coronary artery plaque severity and stenosis detected by CT coronary angiography in asymptomatic Chinese subjects. Int J Cardiovasc Imaging. 2011; 27(5): 669-678, doi: $10.1007 /$ s10554-011-9874-6, indexed in Pubmed: 21695485.

17. Koenig W, Ridker PM. Rosuvastatin for primary prevention in patients with European systematic coronary risk evaluation risk $\geq 5 \%$ or Framingham risk $>20 \%$ : post hoc analyses of the JUPITER trial requested by European health authorities. Eur Heart J. 2011; 32(1): 75-83, doi: 10.1093/eurheartj/ehq370, indexed in Pubmed: 20971747. 